\title{
Radio observations of HD 93129A: The earliest 0 star with the highest mass loss?
}

\author{
P. Benaglia ${ }^{1,2, \star}$ and B. Koribalski ${ }^{3}$ \\ ${ }^{1}$ Instituto Argentino de Radioastronomía, C.C. 5, 1894 Villa Elisa, Argentina \\ 2 Facultad de Cs. Astronómicas y Geofísicas, UNLP, Paseo del Bosque S/N, 1900, La Plata, Argentina \\ 3 Australia Telescope National Facility, CSIRO, PO Box 76, Epping, NSW 1710, Australia \\ Received 31 July 2003 / Accepted 6 November 2003
}

\begin{abstract}
We present the results of radio continuum observations towards the open cluster $\operatorname{Tr} 14$, where our main targets are the early-type O stars HD 93129A/B and HD 93128. The observations were carried out at $3 \mathrm{~cm}(8.64 \mathrm{GHz})$ and $6 \mathrm{~cm}(4.80 \mathrm{GHz})$ with the Australia Telescope Compact Array. Only HD 93129A (type O2 If*) was detected; we measure flux densities of $S_{3 \mathrm{~cm}}=2.0 \pm 0.2 \mathrm{mJy}$ and $S_{6 \mathrm{~cm}}=4.1 \pm 0.4 \mathrm{mJy}$. The resulting spectral index of $\alpha=-1.2 \pm 0.3\left(S_{v} \propto v^{\alpha}\right)$ indicates predominantly non-thermal emission, suggesting HD 93129A may be a binary system. We propose that the observed $3 \mathrm{~cm}$ radio emission is mostly coming from the non-thermal wind collision region of a binary, and, to a lesser extent, from the thermal winds of the primary and secondary stars in HD 93129A. At a stellar distance of $2.8 \mathrm{kpc}$, we derive a mass-loss rate $\dot{M}=5.1 \times 10^{-5} M_{\odot} \mathrm{yr}^{-1}$, assuming the thermal fraction of the $3 \mathrm{~cm}$ emission is $\sim 0.5$.
\end{abstract}

Key words. stars: individual: HD 93129A, HD 93129B, HD 93128 - stars: mass-loss - stars: winds, outflows radio continuum: stars

\section{Introduction}

Massive, early-type $\mathrm{O}$ stars at the upper end of the stellar main sequence are known to have strong stellar winds, with velocities up to $\sim 3000 \mathrm{~km} \mathrm{~s}^{-1}$ (Prinja et al. 1990). Their evolution into Wolf-Rayet (WR) stars is marked by high mass loss rates of $10^{-7}$ to $10^{-5} M_{\odot} \mathrm{yr}^{-1}$ (e.g., Lamers \& Leitherer 1993). The transition can be described by three Of phases $(\mathrm{O}-\mathrm{O}((\mathrm{f}))$ $\mathrm{O}(\mathrm{f})$ - Of - WR), where "f" stands for the presence of certain N III and He II spectral lines (see Maíz Apellániz \& Walborn 2002 for details; also Table 1). Once the H-rich outer layers of the star are lost, the high temperature core is exposed, and the evolution from Of to WR star is complete. The action of the stellar wind on the ambient interstellar medium creates a cavity of hot, rarefied gas, surrounded by a slowly expanding envelope (Benaglia \& Cappa 1999).

Wolf-Rayet stars have anomalously strong and broad emission lines, which are thought to come from the rapidly expanding stellar wind, but few absorption lines. With an average mass of $10 M_{\odot}$, their mass loss rate can reach values up to $10^{-4} M_{\odot} \mathrm{yr}^{-1}$. Van der Hucht et al. $(2001,2002)$ cataloged well over 200 WR stars in our Galaxy, more than half of which belong to the WN class, i.e. they show strong helium and nitrogen emission lines. A large fraction of the cataloged WR stars $(\sim 40 \%)$ are known to be binaries.

Send offprint requests to: $\mathrm{P}$. Benaglia, e-mail: paula@lilen.fcaglp.unlp.edu.ar

* Member of Carrera del Investigador, CONICET.
According to Crowther et al. (1995), the most massive $\left(>60 M_{\odot}\right)$ O stars evolve from the Of stage directly into highly luminous late $\mathrm{WN}(+\mathrm{abs})$ and $\mathrm{WN} 7$ stars without passing through an intermediate Luminous Blue Variable or red supergiant phase. The slightly less massive $\mathrm{O}$ stars evolve through either of those intermediate phases into slightly less luminous WN8 stars (see also Langer et al. 1994). The quantitative spectroscopic properties show a smooth progression from Of stars to lower excitation or late WN stars (WNL) (Bohannan 1990). Walborn et al. (1992), Lamers \& Leitherer (1993) among others, suggested that transition types Ofpe/WNL, and WNL stars, form a sequence of increasing wind density.

Examples of transition objects are Sk-67 22 (type O3If*/WN6-A, Walborn 1982), Sk-71 34 (type O4f/WN3, Conti \& Garmany 1983), and, lately, the ones found in 30 Doradus (Massey \& Hunter 1998). The mass loss rates of the transition objects are expected to lie between those for Of and WR stars.

Almost 400 Galactic $\mathrm{O}$ stars have recently been compiled in the new "Galactic O Star Catalogue" (GOS) by Maíz-Apellániz $\&$ Walborn (2002). There are only about a dozen stars with spectral types $\mathrm{O} 3.5$ or earlier; five of these belong to the clusters Trumpler 14 and Trumpler 16, in the Carina region. Among them one star, HD 93129A, stands out as the earliest, most luminous, and most massive star known in our Galaxy. Walborn et al. (2002) re-analyzed spectra of HD 93129A, previously classified as $\mathrm{O} 3$, and find that it is the prototype of a new subcategory and the sole representative of type $\mathrm{O} 2 \mathrm{If}^{*}$ 
Table 1. Stellar parameters adopted for the target stars.

\begin{tabular}{lrrrl}
\hline \hline & HD 93129A & HD 93129B & HD 93128 & \\
\hline$\alpha, \delta(\mathrm{J} 2000)$ & $104357.462^{a}$ & $104357.638^{b}$ & $104354.372^{c}$ & $\mathrm{~h} \mathrm{~m} \mathrm{~s}$ \\
& -593251.27 & -593253.50 & -593257.37 & $\circ{ }^{\prime \prime \prime}$ \\
Spectral Class. & $\mathrm{O} 2 \mathrm{If}^{* d}$ & $\mathrm{O} 3.5 \mathrm{~V}((\mathrm{f}+))^{d}$ & $\mathrm{O} 3.5 \mathrm{~V}((\mathrm{f}+))^{d}$ & \\
$v_{\infty}$ & $3200 \pm 200^{e}$ & $3070^{f}$ & $3070^{f}$ & $\mathrm{~km} \mathrm{~s}^{-1}$ \\
$T_{\text {eff }}$ & $52000 \pm 1000^{e}$ & $52000 \pm 1500^{g}$ & $52000 \pm 1500^{h}$ & $\mathrm{~K}$ \\
$\log \left(L / L_{\odot}\right)$ & $6.4 \pm 0.1^{e}$ & $5.6^{g}$ & $5.7^{g}$ & \\
\hline
\end{tabular}

References: ${ }^{a}$ Hog et al. (2000), ${ }^{b}$ Worley \& Douglass (1997), ${ }^{c}$ see Maíz Apellániz \& Walborn (2002), ${ }^{d}$ Walborn et al. (2002) (f* means the N IV $4058 \AA$ emission lines are stronger than the N III $4640 \AA$ emission lines, and He II $4686 \AA$ is observed in emission, while ((f+)) means weak N III 4634-4640-4642 A emission lines, strong He II $4686 \AA$ absorption lines, plus Si IV $4089-4116 \AA$ emission lines); ${ }^{e}$ Taresch et al. (1997), ${ }^{f}$ interpolated from Prinja et al. (1990), ${ }^{g}$ see text, ${ }^{h}$ Vacca et al. (1996).

(see Table 1). HD 93129A appears to have the most extreme stellar winds regarding ionization and terminal velocities (Walborn et al. 2002): $v_{\infty}=3200 \pm 200 \mathrm{~km} \mathrm{~s}^{-1}$, an effective temperature $T_{\text {eff }}=52000 \pm 1000 \mathrm{~K}$, and a stellar mass of $130 \pm 15 M_{\odot}$ (Taresch et al. 1997). Only 2.6" from HD 93129A, Walborn (1973) discovered a second star, HD 93129B, of type O3.5 V((f+)). Another star of the same type, HD 93128, is found at a projected distance of $\sim 20^{\prime \prime}$. The stellar parameters for all three stars are listed in Table 1.

In the radio regime, free-free thermal emission from the ionized wind of early-type OB and Wolf-Rayet stars can be modeled (Wright \& Barlow 1975; Panagia \& Felli 1975) yielding a spectral index $(\alpha)$ of typically $\sim 0.6-0.7$. Generally, the radio flux densities are of the order of $\mathrm{mJy}$ for stars at distances up to 2 kpc. About 80 WR stars (Abbott et al. 1986; Chapman et al. 1999) and 120 O stars (Bieging et al. 1989; Scuderi et al. 1998; Benaglia et al. 2001b, etc.) have been observed. Roughly half of them were detected at least at one frequency. When spectral indices could be measured, 40\% showed nonthermal or composite indices.

In recent years the hypothesis was proposed (Chapman et al. 1999; Dougherty \& Williams 2000) that non-thermal emission could be indicative of binarity. In that case, part of the emission comes from the colliding wind region (CWR) of two early massive stars. Chapman et al. (1999) observed 36 WR stars with the ATCA at 3, 6, 13 and $20 \mathrm{~cm}$, obtaining an angular resolution of $\sim 1^{\prime \prime}$ at $3 \mathrm{~cm}$. Dougherty \& Williams (2000) discussed the spectral index behaviour of 25 WR stars, by taking into account observations carried out mainly with the VLA, ATCA, MERLIN and the WSRT. Benaglia \& Romero (2003) presented a table with the characteristics of the nonthermal or composite WR emitters.

Unfortunately, in some particular cases where the nonthermal emission is strong, not enough spectroscopic data are currently available to investigate the binary status. For example, CD-47 4551 (CPD-47 2963), an O4 III(f) star (Walborn 1982), was observed with the ATCA (Benaglia et al. 2001b) and detected with flux densities of $1.77 \pm 0.05 \mathrm{mJy}$ at $3 \mathrm{~cm}$ and $2.98 \pm 0.05 \mathrm{mJy}$ at $6 \mathrm{~cm}(\alpha=-0.89 \pm 0.06)$. If the star is a binary with another WR or OB star, the non-thermal emission could be produced at the CWR of both stars.

Non-thermal radio emission can also be produced by the stellar wind of a single star, as described by White (1985).
In his model, electrons are accelerated to relativistic energies by shocks in the wind near the star, and emit radio radiation. For strong shocks, the expected spectral index is +0.5 . Though this is similar to the thermal index value, the nonthermal flux density coming from a single stellar wind will suffer a break at a frequency of a few $\mathrm{GHz}$, and behave as $S(v) \propto \log (v)$ for larger frequencies, while the thermal flux will obey a $\alpha=0.6-0.7$ law up to the infrared range.

Radio observations are very useful to derive the mass loss rates of early-type stars (Leitherer et al. 1995). The source HD 93129A represents one of the most interesting candidates because it is the only known example of the hottest supergiant class. With the help of high resolution radio data, properties of the wind can be unveiled. In detecting the stellar wind, we intend to identify its radio spectrum, look for a possible colliding wind region, and compare the mass loss rate with those derived from other methods. The observations will also allow us the study of other early-type stars in the region.

The paper is organized as follows: Sect. 2 summarizes the characteristics of the target sources relevant to this study. Section 3 describes the observations and data reduction. In Sect. 4 we present the results, followed by the discussion in Sect. 5. Section 6 contains our conclusions.

\section{The target stars in Trumpler 14}

The field of view of the present data (see Sect. 3) corresponds to the cluster Trumpler 14. Lists of the catalogued members can be found in Massey \& Johnson (1993), and recently DeGioia-Eastwood et al. (2001). A wide range of spectroscopic or photometric distances can be found for $\operatorname{Tr} 14$ in the literature. Humphreys \& McElroy (1984) derived a distance of $3.47 \mathrm{kpc}$. Morrell et al. (1988) discussed two possible distances, 2.8 (variable $R$, from Tapia et al. 1988) and $3.45 \mathrm{kpc}(R=3.2)$, depending on the value taken for the ratio of total to selective absorption $R$. Massey \& Johnson (1993) and Vázquez et al. (1996) found similar values of 3.2 and $3.1 \mathrm{kpc}$, respectively. Most recently, Tapia et al. (2003) computed spectroscopic parallaxes to derive a distance of $2.8 \mathrm{kpc}$. We adopt the latter value throughout this paper. At this distance, the projected cluster size of $140^{\prime \prime}$ (Tapia et al. 2003) corresponds to $1.9 \mathrm{pc}$. Chandra observations towards the $\eta$ Carina region (Evans et al. 2003) revealed strong X-ray emission from the stars HD 93129A/B. Their combined X-ray luminosity is 
$L_{\mathrm{X}}(0.5-2.4 \mathrm{keV})=2.9 \times 10^{33} \mathrm{erg} \mathrm{s}^{-1}$, adjusted to a distance of $2.8 \mathrm{kpc}$.

Penny et al. (1993) presented radial velocity measurements for seven stars in $\operatorname{Tr} 14$, with mean values of $-4.4 \pm$ $7.4 \mathrm{~km} \mathrm{~s}^{-1}$ for HD 93129A, $7.2 \pm 2.4 \mathrm{~km} \mathrm{~s}^{-1}$ for HD 93129B and $7.3 \pm 3.3 \mathrm{~km} \mathrm{~s}^{-1}$ for HD 93128. They concluded that none of them is a strong binary candidate (see also Conti et al. 1977, 1979). Based on the standard deviation in the mean stellar radial velocities, they estimated that the typical random motions in this cluster are $8.5 \mathrm{~km} \mathrm{~s}^{-1}$. For HD 93129A Penny et al. placed an upper limit of $\sim 10 \mathrm{~km} \mathrm{~s}^{-1}$ on the semiamplitude of any short-period binary. To search for possible longperiod binaries they obtained speckle observations, with negative results. Surprisingly, recent HST observations indicate that HD 93129A is a binary (Walborn 2002) with a separation of 55 mas (Walborn 2003, priv. comm.; Nelan et al., in prep.), which could have been detected by Penny et al. (1993).

The optical positions of the three earliest stars in the observed field were taken from the GOS catalog, and come from three different sources, varying in precision: the Tycho-2 cata$\log$ (Hog et al. 2000) lists a very accurate position ( $\sigma \sim 5$ mas) for HD 93129A, whereas the position of HD 93129B originates from the Washington Double Star catalog (Worley \& Douglass 1997) and that of HD 93128 was measured from NTT data, according to Maíz-Apellániz \& Walborn (2002). For a summary of the stellar parameters see Table 1.

HD 93129A. The spectrum of this early $\mathrm{O} 2$ If* star is very similar to Wolf-Rayet stars of spectral type WN6 or WN7 (Walborn et al. 2002). Taresch et al. (1997) studied the spectrum from optical to FUV range, concluding that its strong wind is radiatively driven. By fitting an $\mathrm{H} \alpha$ profile they obtained a mass loss rate of $1.8 \times 10^{-5} M_{\odot} \mathrm{yr}^{-1}$ compared to $2.2 \times 10^{-5} M_{\odot} \mathrm{yr}^{-1}$ derived from $\mathrm{H} \alpha$ measurements by Puls et al. (1996; assuming $\left.T_{\text {eff }}=50500 \mathrm{~K}\right)$.

HD 93129B and HD93128. The effective temperature of HD 93128 is $52000 \pm 1500 \mathrm{~K}$ (Vacca et al. 1996). For HD 93129B, which has the same spectral type as HD 93128, we adopt the same value. The stellar luminosities were derived taking the absolute visual magnitudes from Walborn et al. (2002).

HD 93250. This star is a member of the $\operatorname{Tr} 16$ cluster, located $\sim 10^{\prime}$ East of HD 93129A/B (well outside our primary beam). Leitherer et al. (1995) reported a $3 \mathrm{~cm}$ flux density of $1.36 \pm 0.17 \mathrm{mJy}$ and a $3 \sigma$ upper limit of $3.57 \mathrm{mJy}$ at $6 \mathrm{~cm}$. Assuming a purely thermal spectrum, a distance of $2.2 \mathrm{kpc}$, a wind velocity of $v_{\infty}=3200 \mathrm{~km} \mathrm{~s}^{-1}$ and an effective temperature of $T_{\text {eff }}=50000 \mathrm{~K}\left(T_{\mathrm{e}}=0.4 T_{\text {eff }}=20000 \mathrm{~K}\right.$ according to Drew 1989) Leitherer et al. derived a mass loss rate of $4.1 \times 10^{-5} M_{\odot} \mathrm{yr}^{-1}$. Considering a re-classified spectral type of O3.5 V((f+)) (Walborn et al. 2002) and a distance of $2.5 \mathrm{kpc}$ to $\operatorname{Tr} 16$ (Tapia et al. 2003) we revise the mass loss rate to $5 \times 10^{-5} M_{\odot} \mathrm{yr}^{-1}$ (see Sect. 4.1).
Table 2. Flux densities (or $3 \sigma$ upper limits) of the target stars.

\begin{tabular}{lcc}
\hline \hline Star & $\begin{array}{c}S_{3 \mathrm{~cm}} \\
(\mathrm{mJy})\end{array}$ & $\begin{array}{c}S_{6 \mathrm{~cm}} \\
(\mathrm{mJy})\end{array}$ \\
\hline HD 93129A & $2.0 \pm 0.2$ & $4.1 \pm 0.4$ \\
HD 93129B & $<0.33$ & $<0.63$ \\
HD 93128 & $<0.33$ & $<0.63$ \\
\hline
\end{tabular}

\section{Observations and data reduction}

The observations presented here were obtained with the Australia Telescope Compact Array (ATCA) in January 2003 using the $6 \mathrm{~B}$ configuration. The target field, which contains three massive Of stars, was observed simultaneously at two frequencies: $8.64 \mathrm{GHz}(3 \mathrm{~cm})$ and $4.80 \mathrm{GHz}(6 \mathrm{~cm})$, with a total bandwidth each of $128 \mathrm{MHz}$ over 32 channels. The maximum baseline was $6 \mathrm{~km}$, resulting in angular resolutions around $1^{\prime \prime}-2^{\prime \prime}$. The size of the primary beam is $10^{\prime}$ at $6 \mathrm{~cm}$ and $5^{\prime}$ at $3 \mathrm{~cm}$. The pointing position was $\alpha, \delta(\mathrm{J} 2000)=10^{\mathrm{h}} 43^{\mathrm{m}} 56^{\mathrm{s}}$, $-59^{\circ} 32^{\prime} 51^{\prime \prime}$, slightly offset from the three stars HD 93129A/B and HD 93128. The field was observed during intervals of 8 to $15 \mathrm{~min}$, depending on the atmospheric phase stability, interleaved with short observations of the nearby phase calibrator 1045-62, and spanning an LST range of $12 \mathrm{~h}$. The total time on the target field was nearly $300 \mathrm{~min}$.

The data were reduced in MIRIAD using standard procedures. The flux density scale was calibrated with observations of the primary calibrator PKS B1934-638, assuming flux densities of $2.84 \mathrm{Jy}$ at $3 \mathrm{~cm}$ and $5.83 \mathrm{Jy}$ at $6 \mathrm{~cm}$ (Reynolds 1994). After data calibration the visibilities were Fourier-transformed using "natural"- and "uniform"-weighting. To overcome confusion due to diffuse emission from extended sources in or towards the observed fields, the shortest baselines were removed. For the same reason "uniform"-weighting resulted in the lowest noise levels: 0.11 and $0.21 \mathrm{mJybeam}^{-1}$ at 3 and $6 \mathrm{~cm}$, respectively. The synthesized beam is $1.1^{\prime \prime} \times 1.0^{\prime \prime}$ at $3 \mathrm{~cm}$ and $1.9^{\prime \prime} \times 1.7^{\prime \prime}$ at $6 \mathrm{~cm}$.

\section{Results}

Figures 1 and 2 show the central part of the resulting 3 and $6 \mathrm{~cm}$ radio continuum images. The optical positions of the three target stars (see Table 1) are marked.

Only one radio source, HD 93129A, is detected. Using the MIRIAD task imfit we fit the position and flux density (of a point source) at both frequencies resulting in $\alpha, \delta(\mathrm{J} 2000)=$ $10^{\mathrm{h}} 43^{\mathrm{m}} 57^{\mathrm{s}} .45,-59^{\circ} 32^{\prime} 51.36^{\prime \prime}, 2.0 \pm 0.2 \mathrm{mJy}$ at $3 \mathrm{~cm}$, and $\alpha, \delta(\mathrm{J} 2000)=10^{\mathrm{h}} 43^{\mathrm{m}} 57^{\mathrm{s}} .47,-59^{\circ} 32^{\prime} 51.23^{\prime \prime}, 4.1 \pm 0.4 \mathrm{mJy}$ at $6 \mathrm{~cm}$. From the 3 and $6 \mathrm{~cm}$ flux densities we derive a spectral index of $\alpha=-1.2 \pm 0.3\left(S_{v} \propto v^{\alpha}\right)$ indicating predominantly nonthermal emission. The measured flux densities for HD 93129A and the $3 \sigma$ upper limits for HD 93129B and HD 93128 are listed in Table 2.

The separation between the fitted 3 and $6 \mathrm{~cm}$ radio continuum positions for HD 93129A is $0.2^{\prime \prime}$, slightly larger than the positional uncertainty (which is approximately the size of the synthesized beam divided by signal-to-noise ratio of the 
Table 3. Additional O-type stars within the observed $3 \mathrm{~cm}$ field.

\begin{tabular}{|c|c|c|c|c|c|}
\hline \multirow[t]{2}{*}{ Name } & \multicolumn{2}{|c|}{$\alpha, \delta(\mathrm{J} 2000)$} & \multirow[t]{2}{*}{ Spectral type } & \multirow{2}{*}{$\begin{array}{r}\dot{M}_{\exp } \\
M_{\odot} \mathrm{yr}^{-1}\end{array}$} & \multirow{2}{*}{$\begin{array}{r}S_{\mathrm{exp}, 3 \mathrm{~cm}}^{\mathrm{T}} \mathrm{mJy} \\
\mathrm{m}\end{array}$} \\
\hline & $(\mathrm{h} \mathrm{m} \mathrm{s})$ & $\left({ }^{\circ \prime \prime \prime}\right)$ & & & \\
\hline $\mathrm{Cl}^{*}$ Trumpler $14 \mathrm{MJ} 165$ & 104355.43 & -593249.8 & $\mathrm{O} 8 \mathrm{~V}^{a}$ & $8.5 \times 10^{-7}$ & 0.010 \\
\hline CPD-58 2620 & 104359.92 & -593225.4 & O6.5 V((f) $)^{b}$ & $1.7 \times 10^{-6}$ & 0.016 \\
\hline $\mathrm{Cl}^{*}$ Trumpler $14 \mathrm{MJ} 150$ & 104353.75 & -593329.0 & O9: $\mathrm{V}^{a}$ & $4.5 \times 10^{-7}$ & 0.005 \\
\hline Cl* Trumpler 14 MJ 127 & 104348.82 & -593324.8 & O9 $\mathrm{V}^{a}$ & $4.5 \times 10^{-7}$ & 0.005 \\
\hline CPD-58 2611 & 104346.69 & -593254.8 & O6 V((f) $)^{b}$ & $2.1 \times 10^{-6}$ & 0.021 \\
\hline HD 93160 & 104407.27 & -593430.8 & $\mathrm{O} 6 \operatorname{III}((\mathrm{f}))^{b}$ & $4.9 \times 10^{-6}$ & 0.064 \\
\hline HD $93161 \mathrm{AB}$ & 104408.90 & -593434.9 & $06.5 \mathrm{~V}(\mathrm{f}))^{b}$ & $1.7 \times 10^{-6}$ & 0.016 \\
\hline LS 1832 & 104406 & -593509.0 & $\mathrm{O}^{c}$ & $2.1 \times 10^{-6}$ & 0.021 \\
\hline
\end{tabular}

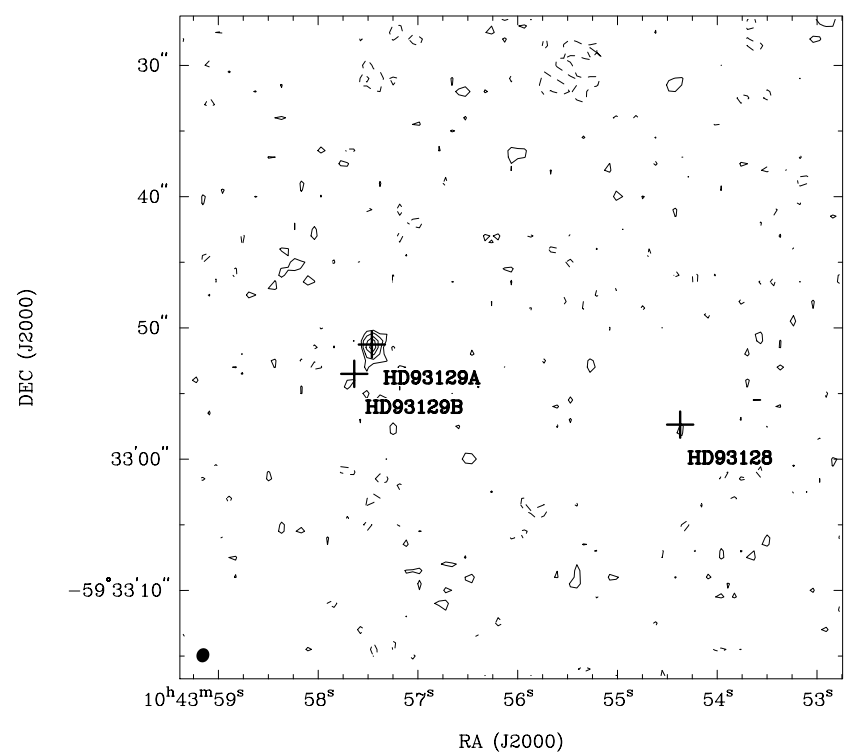

Fig. 1. ATCA $3 \mathrm{~cm}$ radio continuum image towards $\operatorname{Tr} 14$. The optical positions of three stars (see Table 1) are marked. The contour levels are $-0.22,0.22(2 \sigma), 0.44,0.66,0.88$, and $1.10 \mathrm{mJy}$ beam $^{-1}$. The synthesized beam $\left(1.1^{\prime \prime} \times 1.0^{\prime \prime}\right)$ is displayed at the bottom left.

source). The radio and optical positions agree within the given uncertainties.

The Simbad database lists 250 Galactic objects within a radius of $2.5^{\prime}$ around our pointing position (see Sect. 3), at least 11 of which are O-type stars, including the three target stars. Table 3 lists the eight additional stars, none of which was detected. The same $3 \sigma$ upper limits apply.

\subsection{Mass loss rates}

According to Wright \& Barlow (1975), the stellar mass loss rate, $\dot{M}$, of an optically thick ionized wind can be expressed in terms of the measured thermal flux density, $S_{v}$, at a frequency $v$, from radio to IR ranges, as:

$\dot{M}=5.32 \times 10^{-4} \frac{S_{v}^{3 / 4} d^{3 / 2} v_{\infty} \mu}{Z \sqrt{\gamma g_{v} v}} \quad M_{\odot} \mathrm{yr}^{-1}$.

Here $d$ is the stellar distance in kpc, $v_{\infty}$ the wind terminal velocity in $\mathrm{km} \mathrm{s}^{-1}, S_{v}$ the thermal flux density in mJy, and $v$ the frequency in $\mathrm{Hz} ; \mu$ stands for the mean molecular weight of

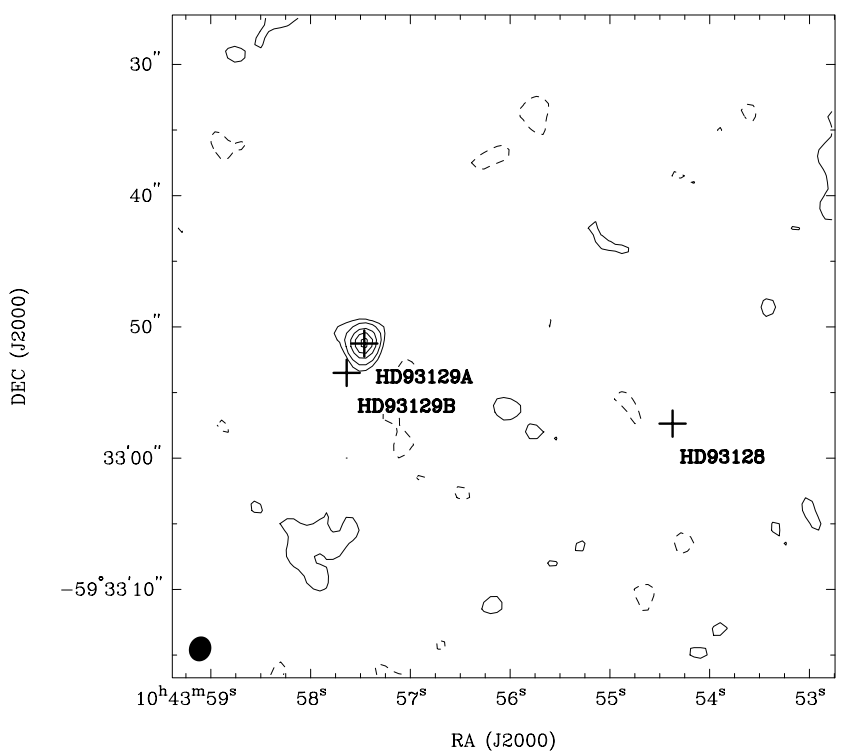

Fig. 2. ATCA $6 \mathrm{~cm}$ radio continuum image towards $\operatorname{Tr} 14$. The optical positions of three stars (see Table 1) are marked. The contour levels are $-0.42,0.42(2 \sigma), 0.84,1.26,1.68$, and $2.10 \mathrm{mJy}$ beam $^{-1}$. The synthesized beam $\left(1.9^{\prime \prime} \times 1.7^{\prime \prime}\right)$ is displayed at the bottom left.

the ions, $\gamma$ is the mean number of electrons per ion and $Z$ the rms ionic charge. For a fully ionized $\mathrm{H}-\mathrm{He}$ gas, $\mu=1.38$. In a first approximation, $Z \approx \gamma \sim 1$. We will take those values for all calculations throughout the text. The Gaunt factor $g_{v}$ can be approximated by:

$g_{v}=9.77\left(1+0.13 \log \frac{\left(0.4 \times T_{\mathrm{eff}}\right)^{3 / 2}}{Z v}\right)$.

From the derived spectral index of $\alpha=-1.2 \pm 0.3$ we know that the observed radio continuum emission consists of thermal and non-thermal components. Their relative contributions (and locations) are unknown since the detected source is essentially unresolved. If $f_{\mathrm{T}}$ is the fraction of the thermal emission at $3 \mathrm{~cm}$ with $S_{3 \mathrm{~cm}}^{\mathrm{T}}=f_{\mathrm{T}} \times S_{3 \mathrm{~cm}}, f_{\mathrm{T}}=0-1$, then the mass loss rate of HD 93129A is $\dot{M}=\left(f_{\mathrm{T}}\right)^{3 / 4} \times 8.6 \times 10^{-5} M_{\odot} \mathrm{yr}^{-1}$. For $f_{\mathrm{T}} \lesssim 1$ this would be one of the highest mass loss rates ever derived for a massive, early-type star. For HD 93129B and HD 93128 we derive $3 \sigma$ upper limits of $\dot{M}<\left(f_{\mathrm{T}}\right)^{3 / 4} \times 2.13 \times 10^{-5} M_{\odot} \mathrm{yr}^{-1}$.

Given a stellar mass, luminosity, effective temperature, and wind terminal velocity, the models of Vink et al. (2000) 
can be used to compute a theoretical - or expected - mass loss rate, $\dot{M}_{\text {exp }}$. Using Vink's program (see astro.imperial.ac.uk/ jvink/) and the values given in Table 1 , we calculate $\dot{M}_{\text {exp }}=2.12 \times 10^{-5} M_{\odot} \mathrm{yr}^{-1}$ for HD 93129A. While this value is in good agreement with the $\mathrm{H} \alpha$-derived mass loss rates (Puls et al. 1996; Taresch et al. 1997), it is a factor four smaller than that derived from the measured $3 \mathrm{~cm}$ radio continuum flux density, suggesting $f_{\mathrm{T}}=$ 0.15 . For HD $93129 \mathrm{~B}$ and HD 93128 we calculate $\dot{M}_{\exp }$ of $0.3 \times 10^{-5} M_{\odot} \mathrm{yr}^{-1}$.

Table 3 lists the expected stellar mass loss rates for the other eight $\mathrm{O}$ stars in the field, estimated as for the earliest stars and assuming a distance of $2.8 \mathrm{kpc}$. The luminosities, masses, and effective temperatures were taken from Vacca et al. (1996). The terminal velocities were interpolated from Table 3 of Prinja et al. (1990) according to spectral type. The expected thermal flux density at $3 \mathrm{~cm}$ was computed inverting Eq. (1), and is listed in the last column.

\section{Discussion}

\subsection{A CWR between HD 93129A and B?}

The distance of $D=2.6^{\prime \prime}=7300$ AU between coordinates of HD 93129A and B is a lower limit to their absolute separation. If a colliding wind region exists between the two stars, it would be located at projected distances $r_{\mathrm{A}}, r_{\mathrm{B}}$ (see Eichler \& Usov 1993):

$r_{\mathrm{A}}=\frac{1}{1+\eta^{1 / 2}} D, \quad r_{\mathrm{B}}=\frac{\eta^{1 / 2}}{1+\eta^{1 / 2}} D$.

The parameter $\eta$ is defined in terms of the wind terminal velocities and the stellar mass loss rates $\dot{M}$ :

$\eta=\frac{\dot{M}_{\mathrm{B}} \times v_{\infty, \mathrm{B}}}{\dot{M}_{\mathrm{A}} \times v_{\infty, \mathrm{A}}}$.

Using the derived theoretical values for $\dot{M}_{\mathrm{A}}$ and $\dot{M}_{\mathrm{B}}$, we find $\eta=0.14$. Thus $r_{\mathrm{A}}=1.9^{\prime \prime} \approx 5350 \mathrm{AU}$, and $r_{\mathrm{B}}=$ $0.7^{\prime \prime} \approx 1950 \mathrm{AU}$. The putative CWR would be centered around $\alpha, \delta(\mathrm{J} 2000)_{\mathrm{CWR}}=10^{\mathrm{h}} 43^{\mathrm{m}} 57.59^{\mathrm{s}},-59^{\circ} 32^{\prime} 52.9^{\prime \prime}$, but no emission was detected with the ATCA. The same $3 \sigma$ upper limits apply (see Table 2).

Assuming a spectral index of $\alpha=-0.5$ (Eichler \& Usov 1993), we derive an upper limit to the non-thermal radio luminosity from a CWR between stars $\mathrm{A}$ and $\mathrm{B}$ of $L_{\mathrm{obs}}<$ $1.4 \times 10^{28} \mathrm{erg} \mathrm{s}^{-1}$. The expected non-thermal luminosity from a possible $\mathrm{A} / \mathrm{B}$ binary is difficult to estimate due to the poor knowledge of system parameters such as size of the CWR and the magnetic field. Moreover, according to Wright \& Barlow (1975), the radii of the radio photospheres at 3 and $6 \mathrm{~cm}$ would be $\sim 30$ and $60 \mathrm{AU},\left(\ll r_{\mathrm{A}}, r_{\mathrm{B}}\right)$, respectively, if $\dot{M}=5 \times 10^{-5} M_{\odot} \mathrm{yr}^{-1}$. The existence of a CWR between HD 93129A and B is very unlikely (see Sect. 5.4).

\subsection{A CWR between the binary components of HD 93129A?}

Walborn (2002) recently reported that HD 93129A is a binary system. Using the HST Fine Guidance Sensor Interferometer,
Nelan et al. (2003, in prep.) resolved it into a 55 mas binary with a magnitude difference of 0.9 mag (Walborn 2003, priv. comm.). In the binary system, we will call "a" the $\mathrm{O} 2$ primary, and "b", the secondary. The total $V$ magnitude measured for HD 93129A is $7.2 \mathrm{mag}$ (Hog et al. 1998). Given that $V_{\mathrm{a}}=7.6 \mathrm{mag}$, and $V_{\mathrm{b}}=8.5 \mathrm{mag}$ (Walborn 2003, priv. comm.), the secondary has a magnitude similar to that of HD 93129B ( $V=8.9 \mathrm{mag}$ ) and HD $93128(V=8.8 \mathrm{mag}$ ) (Walborn et al. 2002). The four stars are probable coeval, and their spectral types should be similar (Nelan et al., in preparation).

The binary separation ( 55 mas $=154$ AU) implies that the stellar winds are likely to interact. In fact, there is a high probability that the system is an $\mathrm{O}+\mathrm{O}$ colliding wind binary. Additional support to this idea can be found by studying the radio spectral index (see Sect. 5.3). Assuming that the companion is an $\mathrm{O} 3.5 \mathrm{~V}$ star with the same terminal velocity, effective temperature, luminosity, and expected mass loss rate than HD 93129B (see Table 1), we find $\eta=0.14, r_{\mathrm{Aa}}=112 \mathrm{AU}$, and $r_{\mathrm{Ab}}=42$ AU. The binary separation of HD 93129A is similar to that of WR 146 (210 AU, Setia Gunawan et al. 2000), thus it is reasonable to assume a similar extent for the CWR, i.e. $\sim 50$ AU. We estimate the equipartition magnetic field for the CWR according to Miley (1980) and find $B_{\mathrm{CWR}} \approx 4 \mathrm{mG}$. Using the magnetic field structure given by Eichler \& Usov (1993), the surface magnetic field of HD 93129Aa can be extrapolated to $B_{*} \sim 100 \mathrm{G}$, if the stellar rotational velocity is $0.1 v_{\infty}$ (Conti $\&$ Ebbets 1997). This estimate is consistent with the few observational values available (Donati et al. 2001, 2002).

Substituting the parameters of HD 93129Aa and Ab into Eqs. (15) and (16) from Eichler \& Usov (1993) we obtain:

$$
L_{\mathrm{rad}} \approx 7.4 \times 10^{31} \beta\left(\frac{\epsilon}{10^{-2}}\right)\left(\frac{\xi}{10^{-3}}\right) \mathrm{erg} \mathrm{s}^{-1}
$$

for the expected non-thermal radio luminosity of the CWR, and $\xi \approx 5 \times 10^{-5}$. Taking typical values of $\beta \sim 0.1, \epsilon \sim 10^{-2}$, we calculate $L_{\mathrm{rad}} \approx 4 \times 10^{29} \mathrm{erg} \mathrm{s}^{-1}$.

From the observed flux densities we obtain a radio luminosity of $L_{\mathrm{obs}}=1.12 \times 10^{29} \mathrm{erg} \mathrm{s}^{-1}$ for HD 93129A between 4.8 and $8.64 \mathrm{GHz}$, which is in good agreement with the total expected one.

In a colliding wind binary system like the one described here, a total X-ray luminosity due to Bremsstrahlung can be computed. Following Usov (1992) we obtain $2.2 \times 10^{33} \mathrm{erg} \mathrm{s}^{-1}$, very close to the observed X-ray luminosity by Evans et al. (2003).

\subsection{Spectral index considerations}

The spectral index of $-1.2 \pm 0.3$ derived from the observations presented here is much steeper than that estimated by White (1985), $\alpha=+0.5$, for synchrotron emission from a single star. Steeper indices can result in the CW scenario as the effect of the softening of the particle spectrum due to inverse Compton (IC) losses (Benaglia \& Romero 2003). At a CWR the accelerated electrons follow a power-law spectrum $N(E) \propto E^{p}$, with $p=-2$. Both synchrotron and IC processes help electrons to lose energy. At high energies, the radiative cooling would be dominated by IC losses, due to the strong photon fields at 
the CWR. At the energy $E_{\mathrm{b}}$ at which the cooling and escape times are equal (e.g. Longair 1997, p. 281), IC losses produce a break in the spectrum, from an index $p$ to $p^{\prime}=p-1$. The same population of relativistic electrons is capable of losing energy via synchrotron emission, producing a flux $S(v) \propto v^{\alpha}$, where $\alpha=(p+1) / 2$. Thus, for $E<E_{\mathrm{b}}, \alpha \approx-0.5$, and for $E>E_{\mathrm{b}}, \alpha \approx-1$. This last value seems to be consistent with the spectral index derived from the present observations. The break energy can be computed if the following set of parameters is known (see Benaglia \& Romero 2003): the mass loss rates, wind terminal velocities, and luminosities of both components, and the magnetic field at the CWR, together with the size $s$ of the non-thermal source. Replacing the values adopted or obtained above, the break frequency in the radio range will be a few GHz. ATCA observations at 1.4 and $2.4 \mathrm{GHz}$ are necessary to test this scenario.

The relative importance of the processes already mentioned can be measured by estimating the time needed for an electron to lose all its energy, either in the form of synchrotron radiation $\left(t_{\text {syn }}\right)$ or IC scattering $\left(t_{\mathrm{IC}}\right)$. The importance of synchrotron and inverse Compton radiation for electrons of the same energy can be measured computing the ratio between the corresponding lifetimes (Longair 1997, p. 274):

$$
\frac{t_{\mathrm{syn}}}{t_{\mathrm{IC}}}=\frac{U_{\mathrm{rad}}}{U_{\mathrm{mag}}},
$$

where $U_{\text {rad }}$ and $U_{\text {mag }}$ are the energy densities of the radiation field and of the magnetic field respectively. Using the observed radio luminosity $L_{\mathrm{obs}}$, we get $U_{\mathrm{rad}}=2.5 \times 10^{-9} \mathrm{eV} \mathrm{m}^{-3}$. The variable $U_{\text {mag }}$ is $6.4 \times 10^{-15} \mathrm{eV} \mathrm{m}^{-3}$. The corresponding time ratio is about $\sim 4 \times 10^{5}$, thus $t_{\text {syn }} \gg t_{\mathrm{IC}}$. Following this last estimate, and under the conditions mentioned above, IC losses should dominate over synchrotron ones.

\subsection{Colliding wind emission from other binaries}

There are only a few examples (WR 146, WR 147, Cyg OB2 No. 5) of early-type binary systems in the literature where radio emission from the CWR has been observed. WR 146 is a WC6+O8 binary (van der Hucht 2001). Dougherty et al. (2000) observed the stars with MERLIN at $5 \mathrm{GHz}$ twice, and with the VLA at $1.4,5,8.5$ and $22 \mathrm{GHz}$. The combination of their MERLIN data with the $22 \mathrm{GHz}$ VLA data allowed them to detect two thermal sources, coincident with the WR and OB stars, plus a non-thermal one in-between, nearer to the OB star, and possibly coming from the CWR. At a distance of $1.25 \mathrm{kpc}$ (van der Hucht et al. 2001), the separation of the stellar components is $\sim 210 \mathrm{AU}$, and the size of the non-thermal source is $\sim 50$ AU (Setia Gunawan et al. 2000). The flux densities quoted in the literature are: $31.4 \pm 0.4 \mathrm{mJy}$ and $28.5 \pm 0.3 \mathrm{mJy}$ at $5 \mathrm{GHz}$ for the source identified as the CWR, $7.0 \pm 1.3 \mathrm{mJy}$ at $22 \mathrm{GHz}$ for the emission possibly related to the WR star, and $10.4 \pm 1.0 \mathrm{mJy}$ from the OB star. The ratios of the non-thermal to thermal flux densities are $\sim 3$ for the $\mathrm{O}$ star, and $\sim 4$ for the WR star.

Contreras \& Rodríguez (1999) presented 8.5 GHz-VLA observations of the star WR 147, a binary system formed by WN8 (h)+B0.5 V components (van der Hucht et al. 2001), at a distance of $650 \mathrm{pc}$ (van der Hucht 2001). They detected a southern, thermal radio source coincident with the WR component, and a northern, non-thermal source. The latter is interpreted as originating at the interaction zone between the winds of the WR and OB stars. Their averaged measured flux densities are $28.4 \pm 0.5 \mathrm{mJy}$ from the thermal source, and $10.4 \pm 0.5$ mJy from the non-thermal source. The stellar separation is $\sim 420$ AU (Setia Gunawan et al. 2001). The non-thermal to thermal flux density ratio is $\sim 0.4$.

Cyg OB2 No. 5 seems to be formed by three stars: an O7 Ia+Ofpe/WN9 contact binary, and a B0 V star in a larger orbit (Contreras et al. 1997; Rauw et al. 1999), located at $1.8 \mathrm{kpc}$ (e.g. Waldron et al. 1998). The separation between the contact pair and the third component is about 1700 AU. Radio observations by Persi et al. (1990) and Miralles et al. (1994) at 1.4, 5, 8.5 and $15 \mathrm{GHz}$, showed that the source coincident with the contact pair presented flux variations, defining a "high" emission state $\left(S_{8.5 \mathrm{GHz}}=5-7 \mathrm{mJy}\right.$, and $\left.\alpha \sim 0\right)$, and a "low" emission state $\left(S_{8.5 \mathrm{GHz}}=1-2 \mathrm{mJy}\right.$, and $\left.\alpha \sim 0.6\right)$. Contreras et al. (1996, 1997) performed VLA observations at 5 and $8.5 \mathrm{GHz}$, detecting a non-thermal source $(\alpha=-2.4 \pm 0.6)$ near the B0 V star, and proposed it was a CWR in the system. They measured a flux density at $8.5 \mathrm{GHz}$ of $0.31 \pm 0.02 \mathrm{mJy}$. The ratio of the emission from the weaker non-thermal source to the emission of the contact pair at the lower state, is about 0.2.

The system HD 93129A presents a binary separation similar to that of WR 146. The ratio of non-thermal to thermal emission is unknown. If the magnetic field at the CWR is equal to the equipartition field derived above, $\sim 4 \mathrm{mG}$, then it is similar to those derived for WR 147 (5 mG) and WR 146 (25 mG); see Benaglia \& Romero (2003). The scarcity of the examples and the huge differences in the ratios of CWR-non-thermal to stellar-thermal flux densities measured for the three cases from the literature, make the results of the comparison inconclusive.

\subsection{The radio spectrum of $H D 93129 A$}

To separate the thermal and non-thermal contributions to the spectrum of HD 93129A, further radio observations are needed at lower and, particularly, at higher radio frequencies. These can be obtained with the ATCA at 1.4, 2.3, 16-25, and 85-91 GHz. The system can be resolved with the Long Baseline Array, but the flux densities of the thermal winds are likely too low for a detection, and an absolute position for the CWR would be difficult to obtain.

If the actual mass loss rate of HD 93129A is $\dot{M}=2.1 \times$ $10^{-5} M_{\odot} \mathrm{yr}^{-1}$, i.e. $f_{\mathrm{T}}=0.15$ (see Sect. 4.1 ), the thermal and non-thermal contributions to the $3 \mathrm{~cm}$ radio flux density are $\sim 0.3$ and $\sim 1.7 \mathrm{mJy}$, respectively. Assuming a spectral index of $\alpha_{\mathrm{T}}=0.6$ for the thermal component, we find the spectral index for the non-thermal component is $\alpha_{\mathrm{NT}} \sim-1.4 \pm 0.3$. The expected total flux densities (see Fig. $3 \mathrm{~b}$ ) are $\sim 1.2, \sim 1$, and $\sim 1.3 \mathrm{mJy}$ at 16,25 and $90 \mathrm{GHz}$, respectively.

In contrast, for $f_{\mathrm{T}}=0.5$ we find $\alpha_{\mathrm{NT}} \sim-2.1 \pm 0.3$, similar to that derived for other Of systems (e.g., Cyg OB2 No. 5, Contreras et al. 1996, 1997). At 16, 25 and $90 \mathrm{GHz}$ we expect $\sim 1.7, \sim 2$, and $\sim 4.1 \mathrm{mJy}$ (see Fig. 3a). In this case, the radio 

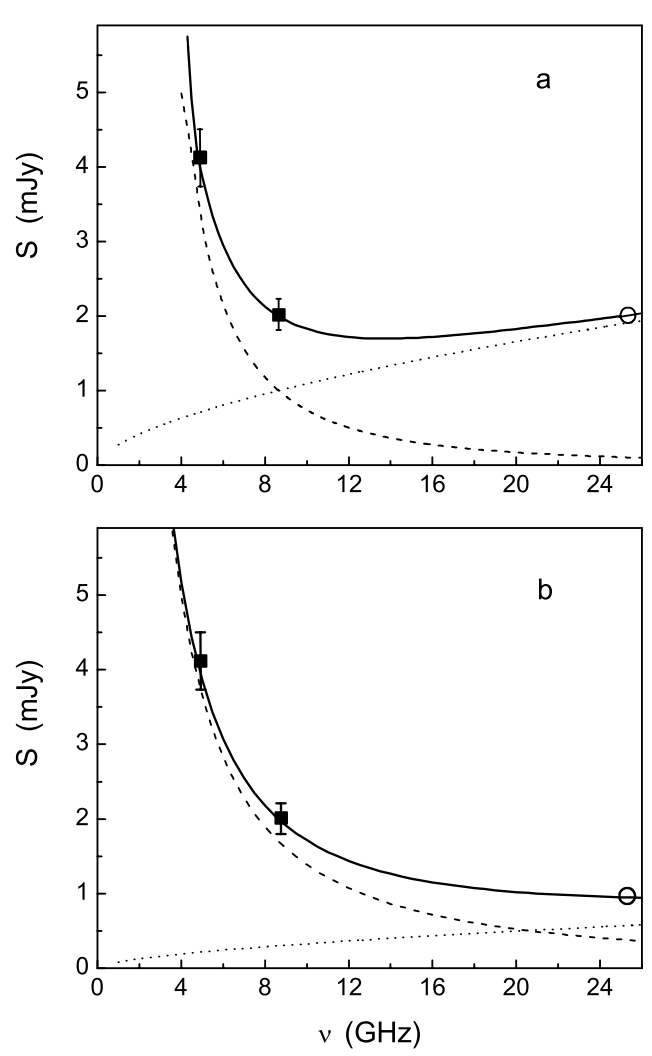

Fig. 3. a) Measured and predicted radio continuum flux densities for HD 93129A in the frequency range from $\sim 1$ to $25 \mathrm{GHz}$. The two measurements at 4.8 and $8.64 \mathrm{GHz}$ are indicated by black squares. Assuming that the flux density measured at $8.64 \mathrm{GHz}, S_{3 \mathrm{~cm}}$, consists to equal parts of thermal and non-thermal emission $\left(f_{\mathrm{T}}=0.5\right)$, and that the thermal emission has a spectral index of $\alpha_{\mathrm{T}}=0.6$, we can fit the observed flux densities by adding a non-thermal component with $\alpha_{\mathrm{NT}}=-2.1 \pm 0.3$. The dotted and dashed lines show the thermal and non-thermal radio emission, respectively. The solid line represents the combined thermal and non-thermal radio emission for this model. b) As Fig. 3a, but assuming $f_{\mathrm{T}}=0.15$. In this case, we can fit the observed flux densities by adding a non-thermal component with $\alpha_{\mathrm{NT}}=-1.4 \pm 0.3$. The total emission expected at $25 \mathrm{GHz}$ is marked with open circles.

derived mass loss rate of the system is $5.1 \times 10^{-5} M_{\odot} \mathrm{yr}^{-1}$. The two scenarios can be distinguished with high-frequency ATCA synthesis observations.

\section{Conclusions}

The field of view towards the Galactic cluster Tr 14 has been observed with the ATCA in the radio continuum at $4.80 \mathrm{GHz}$ $(6 \mathrm{~cm})$ and $8.64 \mathrm{GHz}(3 \mathrm{~cm})$. Only one source, the $\mathrm{O} 2$ If* star HD 93129A, was detected with flux densities of $2.0 \pm 0.2 \mathrm{mJy}$ at $3 \mathrm{~cm}$, and $4.1 \pm 0.4 \mathrm{mJy}$ at $6 \mathrm{~cm}$, resulting in a very steep spectral index of $\alpha=-1.2 \pm 0.3$. This star, recently reported as binary, is the earliest known O-star and potentially the most massive system in the Galaxy, followed by $\eta$ Carina. The observed radio emission is most likely coming from (a) the thermal winds of both binary components and (b) the non-thermal emission from the colliding wind region. The negative spectral index of HD 93129A suggests that the latter is the dominant emission mechanism.

We derive a mass loss rate of $\left(f_{\mathrm{T}}\right)^{3 / 4} \times 8.6 \times 10^{-5} M_{\odot} \mathrm{yr}^{-1}$ for HD 93129A and $3 \sigma$ upper limits of $\left(f_{\mathrm{T}}\right)^{3 / 4} \times 2.13 \times$ $10^{-5} M_{\odot} \mathrm{yr}^{-1}$ for HD 93129B and HD 93128, where $f_{\mathrm{T}}$ is the thermal fraction of the $3 \mathrm{~cm}$ radio continuum emission.

Although the secondary star in the binary HD 93129A remains yet unclassified, the presence of intense non-thermal emission and high radio luminosity together suggest it is another massive, early-type star.

The lack of radio emission near HD 93129B, and the extremely large separation of HD 93129A/B (7300 AU at $2.8 \mathrm{kpc})$ conspires against the existence of a colliding wind region inbetween the stars.

It is crucial to perform observations (i) at lower frequencies, where the thermal contributions should be negligible, and an accurate non-thermal index can be derived; this would help to investigate the processes responsible for the radiation detected; and (ii) at high frequencies, at which non-thermal emission decreases, and the estimation of the thermal flux will enable us to derive the value of the true mass loss rate of HD 93129A.

Acknowledgements. We would like to thank the anonymous referee for comments and suggestions that help to improve the presentation of our investigation. We are indebted to E. Nelan and N. A. Walborn for allowing us to share their HST results before publishing, and to N. A. Walborn and G. E. Romero for a critical reading of the manuscript. P.B. is grateful to the ATNF staff at Sydney and Narrabri. This research has been supported mainly by Fundación Antorchas (P.B.), and has made use of the SIMBAD database operated at CDS, Strasbourg, France.

\section{References}

Abbott, D. C., Bieging, J. H., \& Churchwell, E. 1986, ApJ, 303, 239 Benaglia, P., \& Cappa, C. E. 1999, A\&A, 346, 979

Benaglia, P., Romero, G. E., Stevens, I. R., \& Torres, D. F. 2001a, A\&A, 366, 605

Benaglia, P., Cappa, C. E., \& Koribalski, B. S. 2001b, A\&A, 372, 952

Benaglia, P., \& Romero, G. E. 2003, A\&A, 399, 1121

Bohannan, B. 1990, in Properties of Hot Luminous Stars, ed. C. D. Garmany (Brigham Young Press), 39

Chapman, J. M., Leitherer, C., Koribalski, B. S., et al. 1999, ApJ, 518, 890

Chlebowski, T., \& Garmany, C. D. 1991, ApJ, 368, 241

Conti, P. S., Leep, E. M., \& Lorre, J. J. 1977, ApJ, 214, 759

Conti, P. S., Niemela, V. S., \& Walborn, N. R. 1979, ApJ, 228, 206

Conti, P. S., \& Garmany, C. D. 1983, PASP, 95, 411

Conti, P. S., \& Ebbets, D. 1997, ApJ, 213, 438

Contreras, M. E., Rodríguez, L. F., Gómez, Y., et al. 1996, ApJ, 469, 329

Contreras, M. E., Rodríguez, L. F., Tapia, M., et al. 1997, ApJ, 488, L153

Contreras, M. E., \& Rodríguez, L. F. 1999, ApJ, 515, 762

Crowther, P. A., Smith, L. J., Hillier, D. J., \& Schmutz, W. 1995, A\&A, 293, 427

DeGioia-Eastwood, K., Throop, H., Walker, G., et al. 2001, ApJ, 549, 578

Donati, J.-F., Wade, G. A., Babel, J., et al. 2001, MNRAS, 326, 1265 
Donati, J.-F., Babel, J., Harries, T. J., et al. 2002, MNRAS, 333, 55

Dougherty, S. M., Williams, P. M., \& Pollaco, D. L. 2000, MNRAS, 316,143

Dougherty, S. M., \& Williams, P. M. 2000, MNRAS, 319, 1005

Drew, J. E. 1989, ApJS, 71, 267

Eichler, D., \& Usov, V. 1993, ApJ, 402, 271

Evans, N. R., Seward, F. D., Kraus, M. I., et al. 2003, ApJ, 589, 509

García, B., Malaroda, S., Levato, H., et al. 1998, PASP, 110, 53

Hog, E., Kuzmin, A., Bastian, U., et al. 1998, A\&A, 335, L65 (Tycho-1)

Hog, E., Fabricius, C., Makarov, V. V., et al. 2000, A\&A, 355, L27 (Tycho-2)

van der Hucht, K. A. 2001, New Astron., 45, 135

van der Hucht, K. A., Setia Gunawan, D. Y. A., Williams, P. M., et al. 2001, in Interacting Winds from Massive Stars, ed. A. F. J. Moffat \& N. St-Louis, ASP Conf. Ser., 165, 267

van der Hucht, K. A. 2002, Ap\&SS, 281, 199

Humphreys, R., \& McElroy, D. B. 1984, ApJ, 284, 565

Lamers, H. J. G. L. M., \& Leitherer, C. 1993, ApJ, 412, 771

Langer, N., Hamann, W.-R., Lennon, M., et al. 1994, A\&A, 290, 819

Leitherer, C., Chapman, J. M., \& Koribalski, B. S. 1995, ApJ, 450, 289

Levato, H., Malaroda, S., Morrell, N., et al. 2000, PASP, 112, 359

Longair, M. S. 1997, High Energy Astrophysics, 2 (Cambridge: Cambridge University Press)

Maíz-Apellániz, J., \& Walborn, N. R. 2002, in A Massive Star Odyssey: from Main Sequence to Supernova, ed. K. A. van der Hucht, A. Herrero, \& C. Esteban, ASP Conf. Ser., 212, 560

Mason, B. D., Gies, D. R., Hartkopf, W. I., et al. 1998, ApJ, 115, 821

Mason, B. D., Wycoff, G. L., Hartkopf, W. I., et al. 2001, AJ, 122, 3466

Massey, P., \& Johnson, J. 1993, AJ, 105, 980

Massey, P., \& Hunter, D. A. 1998, ApJ, 493, 180

Miley, G. K. 1980, ARA\&A, 18, 165

Miralles, M. P., Rodríguez, L. F., Tapia, M., et al. 1994, A\&A, 282, 547
Morrell, N., García, B., \& Levato, H. 1988, PASP, 100, 1431

Panagia, N., \& Felli, M. 1975, A\&A, 39, 1

Penny, L. R., Gies, D. R., Hartkopf, W. I., et al. 1993, PASP, 105, 588

Persi, P., Tapia, M., Rodríguez, L. F., et al. 1990, A\&A, 240, 93

Prinja, R. K., Barlow, M. J., \& Howarth, I. D. 1990, ApJ, 361, 607

Puls, J., Kudritzki, R. P., Herrero, A., et al. 1996, A\&A, 305, 171

Rauw, G., Vreux, J.-M., \& Bohannan, B. 1999, ApJ, 517, 416

Reed, B. C., \& Beatty, A. E. 1995, ApJS, 97, 189

Reynolds, J. 1994, A Revised Flux Scale for the AT Compact Array, ATNF Internal Report, AT/39.3/040

Scuderi, S., Panagia, N., Stanghellini, C., et al. 1998, A\&A, 332, 251

Setia Gunawan, D. Y. A., de Bruyn, A. G., van der Hucht, K. A., \& Williams, P. M. 2000, A\&A, 356, 676

Setia Gunawan, D. Y. A., van der Hucht, K. A., Williams, P. M., et al. 2001, A\&A, 376, 460

Simon, K. P., Jonas, G., Kudritzki, R. P., et al. 1983, A\&A, 125, 34

Tapia, M., Roth, M., Vázquez, R. A., et al. 2003, MNRAS, 339, 44

Taresch, G., Kudritzki, R. P., Hurwitz, M., et al. 1997, A\&A, 321, 531

Usov, V. 1992, ApJ, 389, 635

Vacca, W. D., Garmany, C. D., \& Schull, J. M. 1996, ApJ, 460, 914

Vázquez, R. A., Baume, G., Feinstein, A., et al. 1996, A\&AS, 116, 75

Vink, J. S., de Koter, A., \& Lamers, H. J. G. L. M. 2000, A\&A, 362, 295

Walborn, N. R. 1973, ApJ, 179, 517

Walborn, N. R. 1982, AJ, 87, 1300

Walborn, N. R. 1992, ApJ, 393, L13

Walborn, N. R. 2002, in A Massive Star Odyssey: from Main Sequence to Supernova, ed. K. A. van der Hucht, A. Herrero, \& C. Esteban, ASP Conf. Ser., 212, 13

Walborn, N. R., Howarth, I. D., Lennon, D. J., et al. 2002, AJ, 123, 2754

Waldron, W. L., Corcoran, M. F., Drake, S. A. \& Smale, A. P. 1998, ApJS, 118, 217

White, R. L. 1985, ApJ, 289, 698

Worley, C. E., \& Douglass, G. G. 1997, A\&AS, 125, 523

Wright, A. E., \& Barlow, M. J. 1975, MNRAS, 170, 41 